\section{Superhuman mice}

\section{By Tracey Baas, Senior Editor}

The paradox of current mouse-based antibody platforms is that the Ig gene manipulations used to generate the mice can limit the animals' ability to produce robust antibody responses. Kymab Ltd. and Regeneron Pharmaceuticals Inc. have now published details about how their newest mouse platforms circumvent these issues and improve antibody diversity and affinity. ${ }^{1-3}$

Past approaches to engineering mice to develop human antibodies relied on the sequential random insertion of human Ig genes and partial or total elimination of the equivalent mouse Ig genes.

As a result of these gene manipulations, animals lacked fully functional immune systems, which limited their ability to produce responses to some antigens. ${ }^{4-6}$ In addition, sequence replacement was limited to about $100 \mathrm{~kb}$, and the transformation process required up to four rounds of embryonic stem-cell manipulations. ${ }^{7}$

Hampered immune system function was thought to be due to inefficient proteinprotein interactions between human constant regions and mouse $\mathrm{B}$ cell receptors and $\mathrm{Fc}$ receptors. ${ }^{8-10}$ The hampered function led to reduced receptor signaling, limited affinity maturation, antibodies switching between $\operatorname{IgM}, \operatorname{IgD}$, $\operatorname{IgG}, \operatorname{Ig} \mathrm{A}$ or IgE subtypes, and inefficient B cell differentiation into mature antibody-secreting plasma B cells.

Limited antibody production can result from insertion of human genes that lack necessary regions for controlling gene expressionsuch as enhancers, switch regions and regulatory elements-for efficient Ig transcription and recombination. ${ }^{11-13}$

\section{Diplomatic immunity}

To boost the efficacy of their mouse platforms, Kymab and Regeneron each independently engineered animals that retained a functional immune system and contained a larger human Ig sequence component than previous transgenic mice.

Regeneron's approach preserved the intact murine constant regions and swapped mouse variable regions for their human equivalents using large bacterial artificial chromosome-based targeting vectors.

The new version included a much more complete replacement of mouse regions. Regeneron engineered mouse embryonic stem cells
(ESCs) that were used to produce mice in which $6 \mathrm{Mb}$ of mouse genomic sequence had been replaced with $1.4 \mathrm{Mb}$ of human genomic sequence.

Regeneron immunized transgenic mice and wild-type mice with the extracellular domain of the human IL-6 receptor (CD126), and both sets of mice mounted strong antibody responses with similar titer ranges and IgG isotypes, suggesting that humoral response and class switching are similar in both types of mice. In short, the immune systems of the mice were similar.

Importantly, wild-type mice produced antibodies that had mouse variable and constant regions, and transgenic mice produced antibodies that had human variable and mouse constant regions.

The lead high-affinity anti-CD126 antibody from the transgenic mice was reformatted by replacement of mouse Ig constant regions with human Ig constant regions to make a fully human antibody. The CD126 mAb, now called sarilumab (REGN8), is in Phase III testing to treat rheumatoid arthritis and Phase II testing for uveitis.

Data were published in the Proceedings of the National Academy of Sciences.

According to Andrew Murphy, SVP of research at Regeneron, "the company's 15 $\mathrm{mAbs}$ discovered in the VelocImmune mice have entered into clinical development and have all progressed very rapidly-in as little as 18 months-which are time frames that significantly surpass industry standards."

Kymab used large bacterial artificial chromosome-based targeting vectors and added the genes encoding the human variable regions to the mouse genome. Rather than deleting mouse sequence, the company generated large chromosomal inversions of the mouse variable regions to block potential interference between the two sets of genes.

In total, Kymab's engineered mouse ESCs contained an extra $2.7 \mathrm{Mb}$ of human variable Ig genomic sequences.

The Kymab team immunized its transgenic mice with a human CD40 ligand (CD40LG; CD40L; CD154) antigen or a Staphylococcus aureus $\alpha$-hemolysin (aHL) antigen. The resulting antibodies were at least as potent as the anti-CD40L humanized antibody $5 \mathrm{C} 8$ or the anti-aHL human antibody KBSA301.

Biogen Idec Inc.'s 5C8 is in Phase I testing to treat systemic lupus erythematosus. Kenta Biotech Ltd.'s KBSA301 is in Phase I/II testing to treat pneumonia and in Phase I testing to treat Staphylococcus infection.

Results were published in Nature Biotechnology. Kymab did not respond to interview requests.

\section{Constant presence}

According to Hans de Haard, CSO of arGEN-X B.V., the real advance in these studies is that new transgenic mouse systems have been generated with a more normal and functional immune system capable 
of introducing somatic hypermutations and producing antibody amounts comparable to those seen in wild-type mice.

"The human immunoglobulin variable sequences are efficiently rearranged, and their linkage to endogenous immunoglobulin mouse constant sequences provides a flawless interplay with the endogenous signaling machinery," said Roland Buelow, CEO of both Recombinant Antibody Technology Ltd. (RAT) and Open Monoclonal Technology Inc. (OMT). "This means that B cell development appears to be normal and class switch with somatic hypermutation is maintained."

RAT produces large recombinant gene loci using artificial bacterial and yeast chromosomes and works with OMT to produce mouse (OmniMouse) and rat (OmniRat and OmniFlic) platforms for human therapeutic antibody discovery.

de Haard said he has looked at the sequences of both sets of antibodies, and "comparison of the sequences of these new leads with antibodies from the first-generation transgenic mouse systems demonstrates to me that these are indeed better affinity matured by the animals' immune system" than previous mouse-derived human antibodies.

arGEN-X uses llamas for human therapeutic antibody discovery. The company's ARGX-110, a human anti-CD70 (CD27L) antibody, and ARGX-111, a human antibody against c-Met proto-oncogene (MET; HGFR), are both in Phase Ib testing for hematologic malignancies and solid tumors.

An unanswered question is whether increasing the number of transgenic human Ig variable regions will generate greater diversity in human therapeutic antibodies.

"It is debatable if inclusion of more, or indeed all, variable genes is desirable or distractive," said Marianne Brüggemann, director of research and head of the antibody development at both RAT and OMT. "For example, is there really an advantage for including over 20 near-identical variable genes, or will this negatively bias immune diversity?"

"In terms of whether these advances will translate to increased numbers of therapeutic antibodies going into clinical trials, that remains to be seen," de Haard told SciBX.

Both Regeneron and Kymab have patented their respective platforms. Last year, Regeneron filed claims in the English Court alleging that Kymab infringes Regeneron's European patent EP1360287, which covers methods to genetically modify a mouse to make antibodies with human variable regions. Kymab has said it will defend on the grounds of noninfringement and invalidity of the patent.

Baas, T. SciBX 7(17); doi:10.1038/scibx.2014.479

Published online May 1, 2014

\section{REFERENCES}

1. Macdonald, L.E. et al. Proc. Natl. Acad. Sci. USA; published online March 25, 2014; doi:10.1073/pnas.1323896111

Contact: Andrew J. Murphy, Regeneron Pharmaceuticals Inc., Tarrytown, N.Y.

e-mail: andrew.murphy@regeneron.com

Contact: George D. Yancopoulos, same affiliation as above e-mail: george@regeneron.com

2. Murphy, A.J. et al. Proc. Natl. Acad. Sci. USA; published online March 25, 2014; doi:10.1073/pnas.1324022111

Contact: Andrew J. Murphy, Regeneron Pharmaceuticals Inc., Tarrytown, N.Y.

e-mail: andrew.murphy@regeneron.com

Contact: George D. Yancopoulos, same affiliation as above e-mail: george@regeneron.com

3. Lee, E.-C. et al. Nat. Biotechnol.; published online March 16, 2014; doi:10.1038/nbt.2825

Contact: Allan Bradley, Kymab Ltd., Cambridge, U.K. e-mail: abradley@kymab.com

4. Jakobovits, A. Adv. Drug. Deliv. Rev. 31, 33-42 (1998)

5. Mendez, M.J. et al. Nat. Genet. 15, 146-156 (1997)

6. Tomizuka, K. et al. Proc. Natl. Acad. Sci. USA 97, 722-727 (2000)

7. Wallace, H.A. et al. Cell 128, 197-209 (2007)

8. Homabach, J. et al. Nature 343, 760-762 (1990)

9. Nimmerjahn, F. \& Ravetch, J.V. Adv. Immunol. 96, 179-204 (2007)

10. Hjelm, F. et al. Scand. J. Immunol. 64, 177-184 (2006)

11. Garrett, F.E. et al. Mol. Cell. Biol. 25, 1511-1525 (2005)

12. Manis, J.P. et al. Mol. Immunol. 39, 753-760 (2003)

13. Pawlitzky, I. et al. J. Immunol. 176, 6839-6851 (2006)

\section{COMPANIES AND INSTITUTIONS MENTIONED}

arGEN-X B.V., Rotterdam, the Netherlands

Biogen Idec Inc. (NASDAQ:BIIB), Weston, Mass.

Kenta Biotech Ltd., Schlieren, Switzerland

Kymab Ltd., Cambridge, U.K.

Open Monoclonal Technology Inc., Palo Alto, Calif.

Recombinant Antibody Technology Ltd., Cambridge, U.K.

Regeneron Pharmaceuticals Inc. (NASDAQ:REGN), Tarrytown, N.Y. 